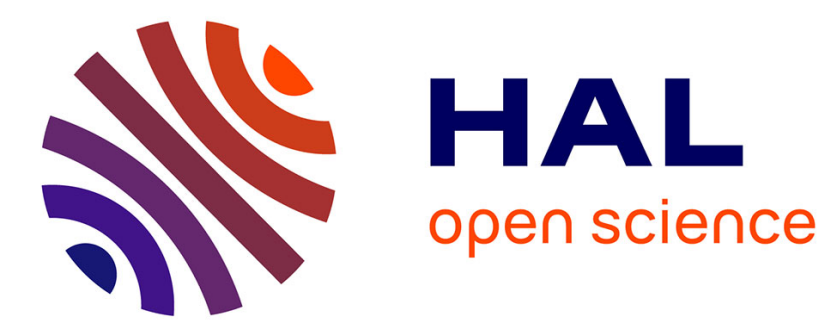

\title{
Design of strain tolerant porous microstructures - A case for controlled imperfection
}

David Jauffres, Christophe L. Martin, Rajendra K Bordia

\section{To cite this version:}

David Jauffres, Christophe L. Martin, Rajendra K Bordia. Design of strain tolerant porous microstructures - A case for controlled imperfection. Acta Materialia, 2018, 148, pp.193 - 201. 10.1016/j.actamat.2017.12.039 . hal-01864382

\section{HAL Id: hal-01864382 \\ https://hal.science/hal-01864382}

Submitted on 8 Jul 2020

HAL is a multi-disciplinary open access archive for the deposit and dissemination of scientific research documents, whether they are published or not. The documents may come from teaching and research institutions in France or abroad, or from public or private research centers.
L'archive ouverte pluridisciplinaire HAL, est destinée au dépôt et à la diffusion de documents scientifiques de niveau recherche, publiés ou non, émanant des établissements d'enseignement et de recherche français ou étrangers, des laboratoires publics ou privés. 


\title{
Design of strain tolerant porous microstructures - A case for controlled imperfection
}

\author{
David Jauffrès ${ }^{a,}{ }^{*}$, Christophe L. Martin ${ }^{a}$, Rajendra K. Bordia ${ }^{b}$ \\ a Univ. Grenoble Alpes, CNRS, Grenoble INP, SIMaP, F-38000 Grenoble, France \\ ${ }^{\mathrm{b}}$ Department of Materials Science and Engineering, Clemson University, Clemson, SC, USA
}

\begin{abstract}
Porous materials, especially ceramics, are used in an ever-expanding range of functional applications. In most cases there are minimum mechanical requirements which limit the porosity level and thus the functional performance provided by the pore surface or volume. In order to design porous materials with the best compromise between functional and mechanical performance, a sound understanding of microstructure-mechanical properties relationships is required. In the current work, discrete simulations are used to assessed the Young's modulus and fracture toughness of various realistic porous microstructures obtained via partial sintering of powders. Scaling laws relating these quantities to microstructural parameters are derived and it is demonstrated that the proportionality between Young's modulus and fracture toughness, often claimed for partially sintered materials, is actually an approximation of a more general relationship. The proposed scaling laws suggest new strategies to build microstructurally tougher and strain tolerant porous materials. It is shown that strain tolerant microstructures can be designed by introducing controlled heterogeneity and hierarchy. Finally, the proposed scaling relationship between Young's modulus and fracture toughness is simplified to give it a practical use and verified for a wide range of porous microstructures, including hierarchical ones.
\end{abstract}

\section{Introduction}

Porosity is an essential component of functional materials for a wide range of applications such as filtration [1,2], tissue engineering [3-5] or electrodes for energy conversion [6-8] and storage [9]. In the above-cited examples, porous ceramics are still mainly obtained by a simple and efficient route, partial sintering, in which powder particles are bonded together by solid necks formed by material diffusion at high temperature. As a way to enhance functional performance, partial sintering can be combined with templating [2,4-7] or additive manufacturing [3] in order to form hierarchical microstructures. For functional applications, the admissible level of porosity is often governed by minimum mechanical strength required for handling, self-support or thermal cycling. If higher levels of porosity could be engineered without compromising the mechanical properties through efficient microstructural design, the functional performance would be enhanced. For partially sintered materials, Young's modulus $E$ and fracture

\footnotetext{
* Corresponding author.

E-mail address: david.jauffres@grenoble-inp.fr (D. Jauffrès).
}

toughness $K_{I c}$ both depend on the average neck size $a_{b}$ and on the average coordination number $Z$ (the number of sintered necks per particle), two metrics conventionally used to describe the microstructure [10-12]. Consequently, as Green and Hardy underlined [11]: "elasticity and fracture toughness of partially-sintered materials are linked in a fundamental way". A sound relationship between these two important material properties and the microstructure should be useful to select promising microstructures and help design optimal porous microstructures for specific applications.

There is a broad class of problems in which the material or component is subjected to internal stresses due to differential strain. A classic example of this is thermal expansion induced stresses - either due to thermal expansion coefficient mismatch (e.g. coatings on substrates) or due to temperature gradients (e.g. thermal shock). Another problem of significant contemporary interest is the cyclic degradation of Li-ion battery electrodes due to strains induced during battery charging and discharging [13-15]. In all of these cases, the ratio $K_{I c} / E$ should be maximized [16]. This parameter can be considered as the "strain tolerance" design parameter. Nonetheless, a large body of experimental data supports 
the existence of a simple proportionality law between $E$ and $K_{I c}$ for porous ceramics [17-20]. Unfortunately, if this proportionality is verified, the ratio $K_{I c} / E$ would be independent of microstructure and porosity, thus leaving no freedom to enhance strain tolerance via microstructural design. However, Green and Hardy [11] suggested, based on previous analytical work by their group [10] and by Arato et al. [12], that the coordination number $Z$ should play a role. The analytical nature of their derivations required strong assumptions such as small bond size and homogeneous strains [10-12]. The validation of the proposed relationships using experimental data was only partial, in particular because $Z$ and $a_{b}$ could not be measured [11].

In the present work, Discrete Element Method (DEM) simulations are used to investigate more deeply the relationships between microstructure, fracture toughness and elasticity of partially sintered materials. DEM allows the generation of a wide range of particulate microstructures with direct access to $Z$ and $a_{b}$ and also access, via appropriate simulations, to mechanical properties [21,22]. DEM results lead us to revisit previous analytical analyses in order to propose scaling laws involving $Z$ and $a_{b}$. Based on these findings, hierarchical heterogeneous microstructures are investigated as strategies to improve the strain tolerance of partially sintered materials.

\section{Methods}

In DEM, the porous material is represented as a packing of overlapping spherical particles obtained numerically via compaction followed by sintering. Appropriate contact laws and bond fracture criteria are used to simulate the mechanical behavior of the microstructure described by the packing. The DEM bond model used here is physically based, does not include any fitting parameter and has been validated by comparison with both Finite Element (FE) analysis and experimental data from the literature $[21,22]$.

\subsection{Sample generation}

Realistic microstructures of partially sintered ceramics are generated by DEM in three steps. First, a gas of particle is built by random sequential addition. Second, the gas is densified up to a given green density, generally in the range $0.5-0.6$. For this step, an hydrostatic pressure is applied while particles interact according to Hertzian contact law [23]. Third, the green microstructure is numerically sintered up to its final density. The sintering model proposed by Bouvard and Mcmeeking [24] was used with parameters typical of ceramics [25].

This procedure allows the generation of heterogeneous and hierarchical microstructures via the use of pore formers or by sintering of already consolidated aggregates. In the first case a second population of larger particles are added during the gas generation and removed before sintering to mimic pore formers (Fig. 1a). In the second case, aggregates with a given size are randomly cut within a microstructure already generated by compaction and sintering, and used to create the initial gas. Then the gas is conventionally compacted and sintered (Fig. 1b).

For this study, the following sets of fully periodic numerical samples were generated:

- Two sets of homogeneous samples with green densities of 0.5 and 0.6 with 100,000 particles of radius $R$ and approximate dimensions of $10 R \times 200 R \times 400 R$ similar to the ones used in Ref. [22].

- A set of heterogeneous and hierarchical samples generated by the pore former technique with a green density of 0.6 within the walls (i.e. microporous material in-between macropores), 200,000 particles and approximate dimensions of $20 R \times 200 R \times$ $400 R$. The pore formers were 5 times larger than the particles and their volume fraction was set to 0.25 (with regard to the volume of ceramic particles). Referred hereafter as pore former samples.

- A set of heterogeneous and hierarchical samples generated by the sintering of pre-calcined aggregates. 150,000 particles were used and the dimensions of the periodic box was approximately $15 R \times 200 R \times 400 R$. Aggregates of density 0.77 from a sintered body with a green density 0.6 were used. The size of the aggregates was 6 times the particle size with a distribution of $\pm 33 \%$. Referred hereafter as aggregate samples.

The large number of particles used is required to correctly assess fracture toughness [22]. For each set of samples, different values of final density are obtained by stopping the course of the numerical sintering at various simulation times.

\subsection{Elasticity modeling}

Contact laws were obtained from FE calculations at the scale of a bond and from beam theory. Normal stiffness, tangent stiffness and rotational stiffnesses of the bond are intricate functions of the elastic properties of the particles (Young's modulus and Poisson ratio) and of the size of the neck [21,26-28]. In addition, a so-called bond interaction displacement is introduced within the normal contact law to account for the elastic ovalization of the spherical particle under load [21,27]. The macroscopic behavior results from writing explicitly the force and moment equilibrium for each particle. The effective elastic properties (Young's modulus and Poisson's ratio) are deduced from the average stresses and strains via the Hooke's law. This elastic model, containing no fitting parameter, has been validated by comparison with both FE and experiments [21]. The main limitation of this modeling approach is its validity at high relative density. Indeed, the model assumptions break down when impingement of sintered necks becomes significant. This typically occurs around $a_{b} / R=0.5$ to 0.6 (corresponding to densities in the range $0.75-0.85$ for homogeneous microstructures).

\subsection{Fracture toughness modeling}

The contact law is complemented with bond fracture criteria leading to the brittle fracture of bonds in tension or shear. An analytical calculation of the strain energy release rate at the scale of a single bond leads to fracture criteria that are functions of the intrinsic bond toughness. In this approach, the neck between the two particles is considered as an advancing crack with a stress singularity [22]. Assuming that the only energy penalty for fracture at the scale of a bond is the creation of free surfaces (no plasticity) the intrinsic bond toughness is approximated as twice the surface energy $\gamma_{s}[22,29]$. The fracture toughness of the numerical microstructures is then evaluated by applying Linear Elastic Fracture Mechanics (LEFM) to the fully periodic slabs equipped with a precrack and strained up to failure [22]. We have shown that this procedure successfully reproduced experimental data on porous alumina, yttria-stabilized zirconia - nickel oxide composite and magnesia - magnesium aluminate composite with a typical surface energy for ceramics of $1 \mathrm{~J} \mathrm{~m}^{-2}$ [22]. These physically motivated bond fracture criteria lead to an Orowan-Petch type relationship between the strength and the inverse square root of particle size [30]. However, fracture toughness is independent of particle size [22]. 
(a)

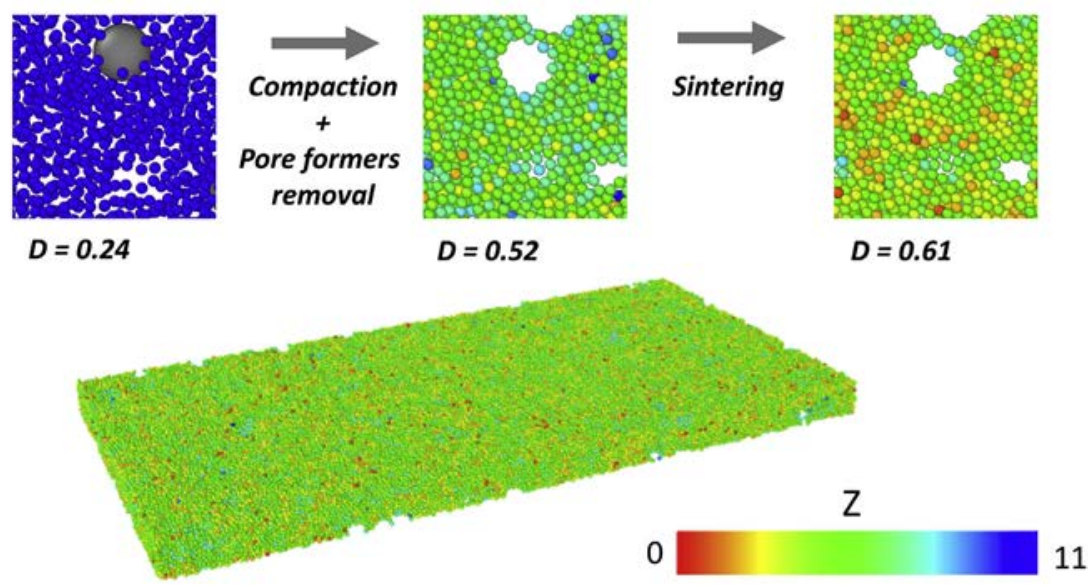

(b)

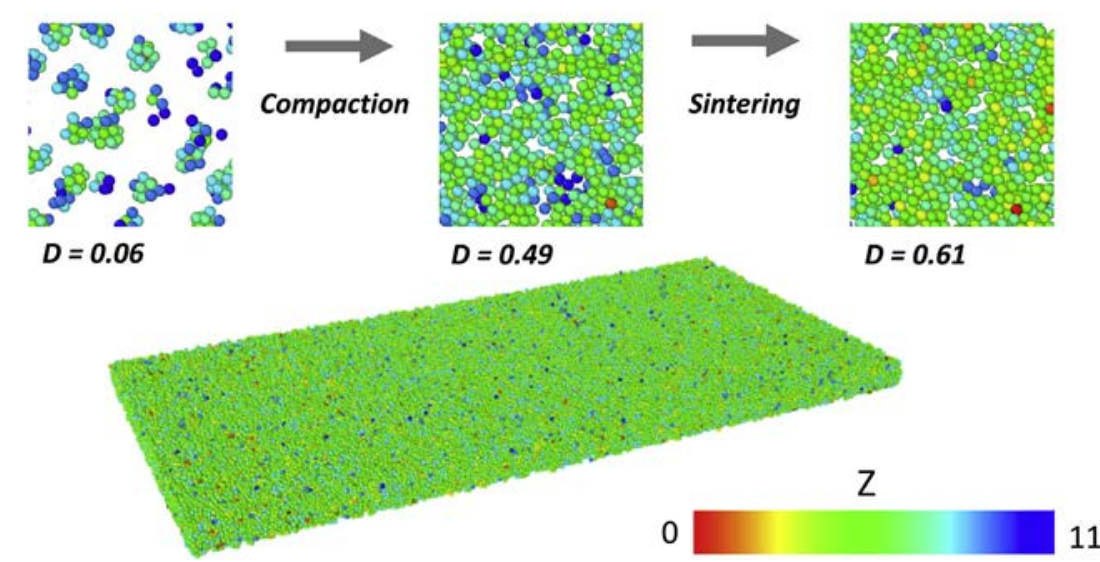

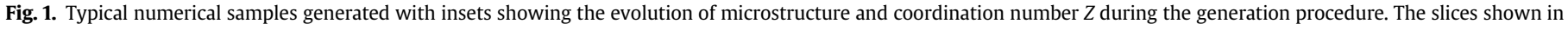
insets are 3 particles thick. (a) Pore former sample. (b) Aggregate sample.

\section{Results and discussion}

\subsection{Homogeneous microstructures}

In order to elucidate the relationships between $E, K_{I C}$ and the microstructure, samples with homogeneous porous microstructures were studied for two different initial green densities of 0.5 and 0.6 (hereafter denominated $D_{0} 50$ and $D_{0} 60$ ), in the final density range of $0.5-0.8$. Two assumptions have been proposed to calculate the neck size [31,32]: (1) the excess matter within the particle overlap region is redistributed homogeneously within the particle (truncated spheres model, Fig. 2a) or (2) redistributed within the neck (tangent torus model) which leads to larger (and thus stiffer) bonds (Fig. 2b). This later geometric approximation was first proposed by Coble [31]. In a real system, the exact redistribution of matter depends on the mass transport mechanisms and their relative importance over the course of sintering [24,31,33]. For example, systems promoting surface diffusion and/or evaporationcondensation (non-densifying mechanisms) via non-conventional sintering [34-36] or nanoparticle addition [37,38] are believed to be closer to the Coble's model while conventionally sintered alumina is probably closer to the truncated spheres model [21]. Indeed, the center-to-center approach of the particles is governed by the density and, at a given density, the necks will be larger if both densifying and non-densifying mechanisms are operating. As reported in Fig. 3, a wide range of neck size and coordination number is obtained with our numerical samples. Note that samples with a lower green density exhibit a larger neck size and a lower coordination number while samples using Coble's model have larger necks but similar coordination compared to samples using truncated spheres model. DEM simulations provide dimensionless Young's modulus $\tilde{E}=\frac{E}{E_{0}}$ and fracture toughness $\tilde{K}_{I c}=K_{I c} / \sqrt{\gamma_{s} \frac{E_{0}}{1-v_{0}^{2}}}$ for each microstructure where $E_{0}, v_{0}$ and $\gamma_{s}$ are the Young's modulus, Poisson's ratio and surface energy of the dense material $[21,22]$. The simulation results are used in the following to assess microstructure-mechanical properties relationships.

Based on an analytical analysis of a sphere subjected to an hydrostatic pressure, Green et al. proposed a scaling relationship between the effective bulk modulus $K$, the average compliance of a contact $\Phi$ and the coordination number $Z: K \propto \frac{Z}{\Phi R}[10]$. However, our DEM simulations did not confirm this relationship, likely due to the simplifying assumptions used by Green et al., in particular the Voigt assumption (i.e. homogeneous strain). Indeed, it has been shown that decreasing the coordination number $Z$ increases the strain field heterogeneity, thus moving the system away from the Voigt assumption [39]. In order to account for this effect, Green's relation is modified using a power exponent $m$ on $Z$, yet to be determined. In addition, since the Poisson's ratio exhibits only limited variations in the considered density range [21], the 
(a)

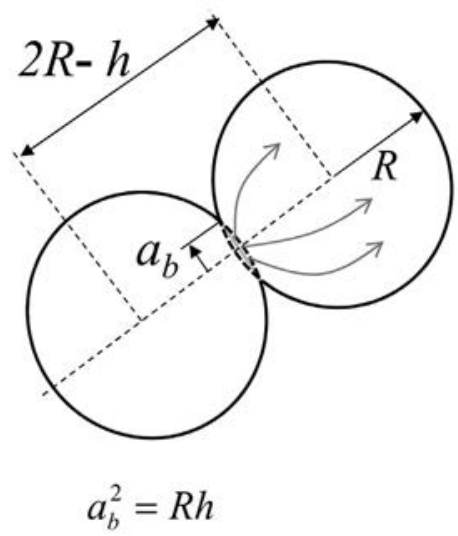

(b)

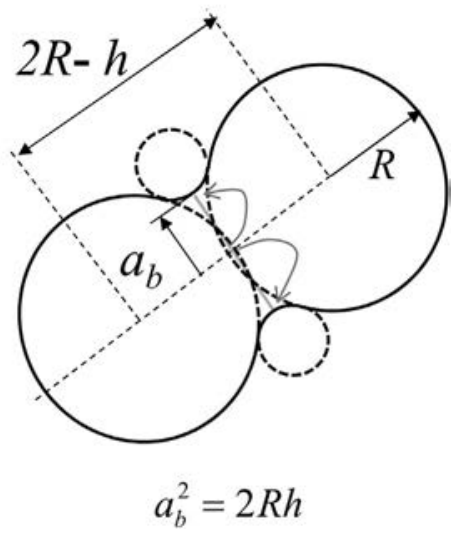

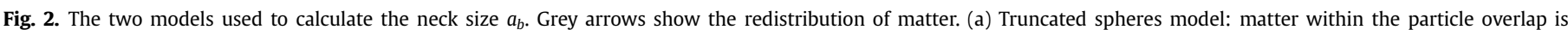
redistributed in volume. (b) Tangent torus model or Coble's model: matter within the particle overlap is redistributed within the neck.

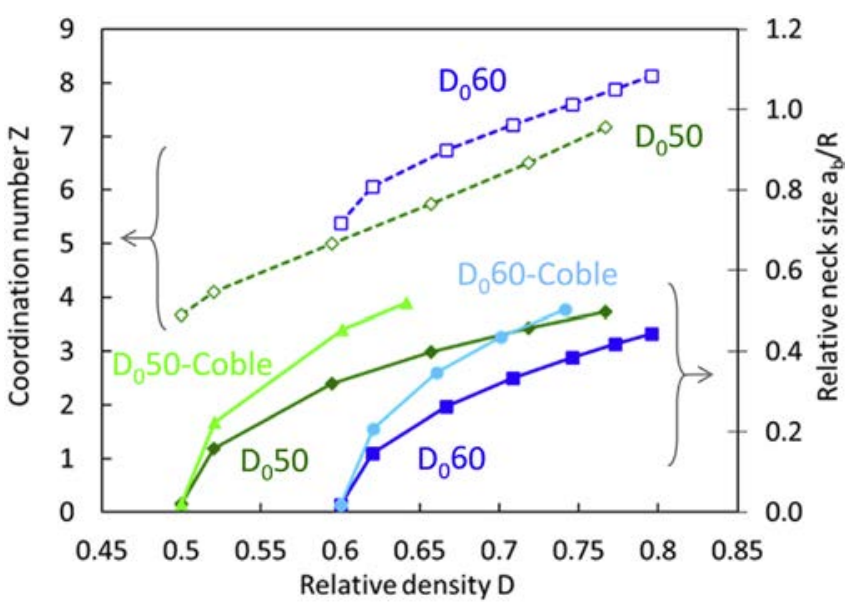

Fig. 3. Evolutions of the coordination number $Z$ and of the relative neck radius $a_{b} / R$ as a function of relative density for homogeneous microstructures. Samples using the truncated spheres model at the necks are referred as $D_{0} 50$ and $D_{0} 60$ for green densities of 0.5 and 0.6 respectively while samples using Coble's model are referred as $D_{0} 50$ Coble and $D_{0} 60$-Coble. $Z$ of $D_{0} 50$-Coble and $D_{0} 60$-Coble are similar to $D_{0} 50$ and $D_{0} 60$ ones respectively and thus are not reported for the sake of clarity. Samples with a lower green density have a larger neck size but a lower Z. Using the Coble assumption leads also to larger neck sizes but without reducing $Z$. (For interpretation of the references to colour in this figure legend, the reader is referred to the Web version of this article.)

relationship can be written in terms of the Young's modulus:

$E \propto \frac{Z^{m}}{\Phi R}$

The compliance of a finite particle junction has been deduced from FE analysis by McMeeking et al. [26,27] and is used in the DEM model [21]. It is a complex function of the relative neck size and of the elastic properties of the particles, including their Poisson ratio $\nu_{0}$. From the actual expression (Eq. (4) in Ref. [21]), the normalized compliance is plotted as a function $a_{b} / R$ in Fig. 4 for $\nu_{0}=0.2$. The best power-law fit to the actual expression in the range $0.1<\frac{a_{b}}{R}<0.5$ gives the following scaling law:

$\Phi R \propto\left(\frac{a_{b}}{R}\right)^{-1.4}$

After substitution in Eq. (1) a scaling law relating Young's

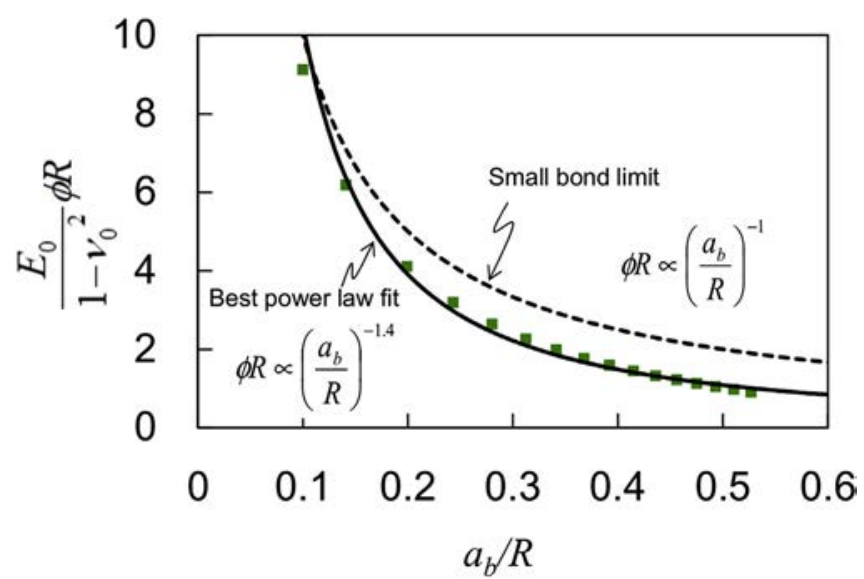

Fig. 4. Compliance of a finite contact according to Eq. (4) in Ref. [21]. The small bond limit corresponding to a Hertzian contact is also shown for completeness.

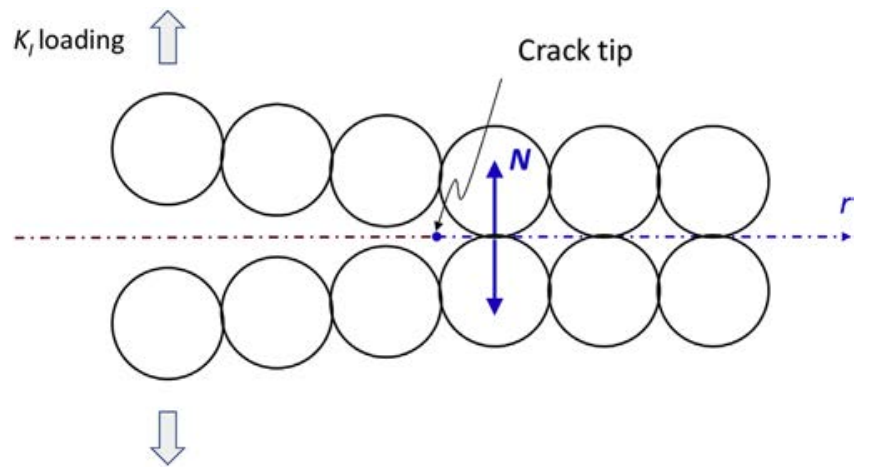

Fig. 5. Force $N$ at the first contact ahead of the crack tip in a regular array of spheres loaded in pure mode I. $r$ is the distance from the crack tip.

modulus to microstructural parameters is obtained:

$E \propto Z^{m}\left(\frac{a_{b}}{R}\right)^{1.4}$

Assuming an elastic-brittle behavior, a similar scaling law can be derived for fracture toughness. A simple analysis on a cubic array of sintered particle equipped with a crack, as sketched in Fig. 5, gives 
the force $N$ at the first contact ahead of the crack tip [11,22]. Briefly, the stress normal to the crack plane is given by LEFM:

$\sigma_{z z}=\frac{K_{I}}{\sqrt{2 \pi r}}$

where $r$ is the distance from the crack tip and $K_{I}$ the mode-I stress intensity factor. This stress can then be integrated to obtain the expression for $N[11,22]$ :

$N=\int_{0}^{2 R} \sigma_{z z} 2 R d r=\frac{4 K_{I} R^{3 / 2}}{\sqrt{\pi}}$

However, for a random geometry, the force $N$ should depend on the coordination number $Z$. It is expected that $N$ decreases with increasing $Z$ as more contacts become available to distribute the mechanical loading. Consequently, and in contrast with Green et al.'s work [11] that did not consider any effect of $Z$ on $K_{I c}$, the more general form is proposed:

$N \propto \frac{K_{I} R^{3 / 2}}{Z^{p}}$

where $p$ is an exponent to be fitted to DEM numerical simulation data. At the onset of failure, the force reaches $\sigma_{f} \pi a_{b}{ }^{2}$ where $\sigma_{f}$ is the strength of the bond leading to the following scaling law for fracture toughness:

$K_{I c} \propto \sigma_{f} \sqrt{R}\left(\frac{a_{b}}{R}\right)^{2} Z^{p}$

The bond strength $\sigma_{f}$ has been obtained as a function of $\Phi, a_{b}$ and the bond toughness $\Gamma$ in Ref. [22], from a strain energy release rate calculation at the scale of the bond that is considered as an advancing crack (a stress singularity is assumed at the tip of the crack):

$\sigma_{f}=2 \sqrt{\frac{\Gamma}{\pi a_{b}^{2} \Phi}} \propto a_{b}{ }^{-0.5}\left(\frac{a_{b}}{R}\right)^{0.2}$

Combining Eqs. (7) and (8) leads to:

$K_{I c} \propto Z^{p}\left(\frac{a_{b}}{R}\right)^{1.7}$

Finally, using a best-fit procedure to the DEM simulation results, the exponents $m$ and $p$ are determined for Eqs. (3) and (9). An alternative approach is to assume for both $E$ and $K_{I c}$ a scaling law of the general form $Z^{m}\left(\frac{a_{b}}{R}\right)^{n}$ and apply a best fit procedure on both exponents $m$ and $n$, without considering the above analytical assessments for $n$. Values for the exponents obtained using the two methods are reported in Table 1. It is worth noting that the exponents obtained from DEM data are very close to those determined using the analytical values for $n$ thus supporting our analysis and procedure. Fig. 6 shows that Eqs. (3) and (9) capture strikingly well the influence of microstructure on Young's modulus and fracture toughness. The proposed scaling laws confirm the intuitive idea of increasing the neck size to improve the mechanical properties at a given density, already demonstrated experimentally by several researchers [34-38]. However, the fracture toughness is markedly less sensitive to $Z$ than the Young's modulus which has important implications when considering a strain tolerant design. The strain tolerance parameter $K_{I c} / E$ can be directly deduced from Eqs. (3) and (9): $\frac{K_{C}}{E} \propto \frac{\left(a_{b} / R\right)^{0.3}}{Z^{0.9}}$. It is clear from this relationship and the corresponding plot (Fig. 7) that the strain tolerance, at a particular value of sintered density, can be significantly improved by starting with a lower green density sample that promotes larger neck size and lower $Z$, but only slightly by promoting surface diffusion that will only increase the neck size and keep $Z$ constant.

\subsection{Hierarchical heterogeneous microstructures}

Additional insights on the effect of the microstructure on strain tolerance are obtained by using DEM on more complex engineered microstructures. In particular, we postulate that the introduction of heterogeneity may have a beneficial effect on strain tolerance. For example, heterogeneity and hierarchy resulting from the use of pore formers to create a population of macropores is beneficial for functionality [2,5-7] and has also proven to be efficient in improving Young's modulus and strength [40]. Another strategy to introduce heterogeneity is the use of pre-calcined aggregated powders [41]. In order to investigate the mechanical performance of heterogeneous and hierarchical microstructures two additional sets of samples were studied:

- pore former samples obtained with spherical pore formers,

- aggregate samples obtained by sintering of pre-calcined aggregates.

The green density within the walls was set to 0.6 for comparison with $D_{0} 60$ homogeneous samples investigated in section 3.1. This choice leads to global green densities of 0.52 and 0.49 for pore former and aggregate samples, respectively. Also, still for the sake of comparison, only the truncated sphere model is considered for the necks. Fig. 8 displays slices of typical 3D microstructures of homogeneous, pore former and aggregate samples at an identical sintered relative density of 0.61 . The mechanical performance of these microstructures is directly assessed using the strain tolerance parameter $\tilde{K}_{I c} / \tilde{E}$ and reported in Fig. 9a. It is clear that the microstructure has a significant effect on this parameter. For example, at a relative density as low as 0.55 , the strain tolerance of a microstructure with hard aggregates is much larger than for a homogeneous microstructure with relative density of the order of $0.7-0.8$. The effect is dramatic in that by introducing suitable heterogeneity, at a given sintered density, the strain tolerance parameter can be increased by as much as a factor of two over comparable homogeneous microstructure. Note that adding spherical macropores using pore formers significantly improves the fracture toughness, but also increases the Young's modulus thus leading only to a slight

Table 1

Scaling laws exponents from best fit procedure.

\begin{tabular}{|c|c|c|c|c|c|}
\hline & \multicolumn{2}{|l|}{$E \propto Z^{m}\left(\frac{a_{b}}{R}\right)^{n}$} & & \multicolumn{2}{|l|}{$K_{I c} \propto Z^{p}\left(\frac{a_{b}}{R}\right)^{q}$} \\
\hline & best fit $m \& n$ & analytical $n \&$ best fit $m$ & & best fit $p \& q$ & analytical $q \&$ best fit $p$ \\
\hline$m$ & 2.4 & 2.4 & $p$ & 1.5 & 1.5 \\
\hline$n$ & 1.3 & $1.4^{\mathrm{a}}$ & $q$ & 1.8 & $1.7^{\mathrm{a}}$ \\
\hline
\end{tabular}

${ }^{a}$ Values given by Eqs. (2) and (7). 
(a)

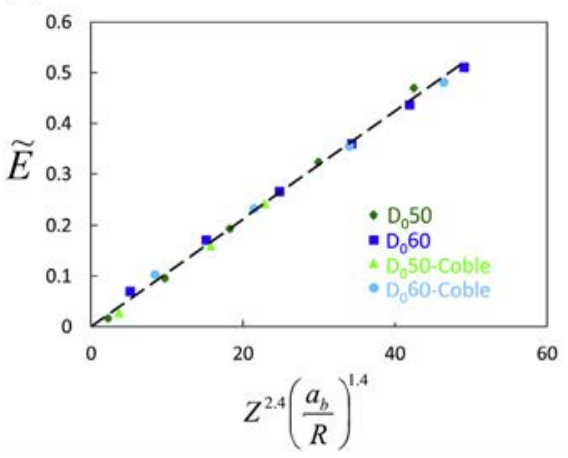

(b)

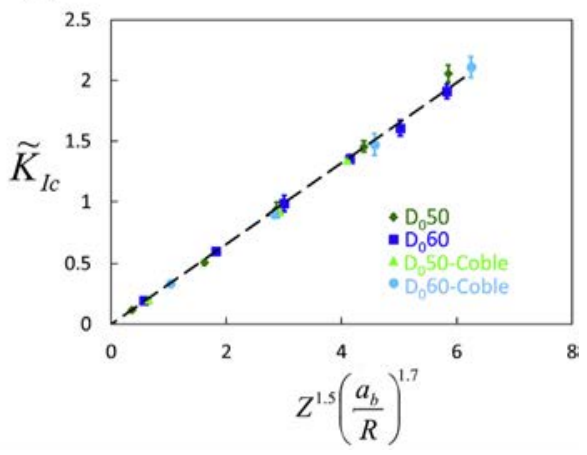

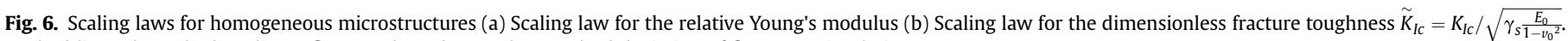
Dashed lines show the best linear fit. Error bars denote the standard deviation of five DEM simulations.

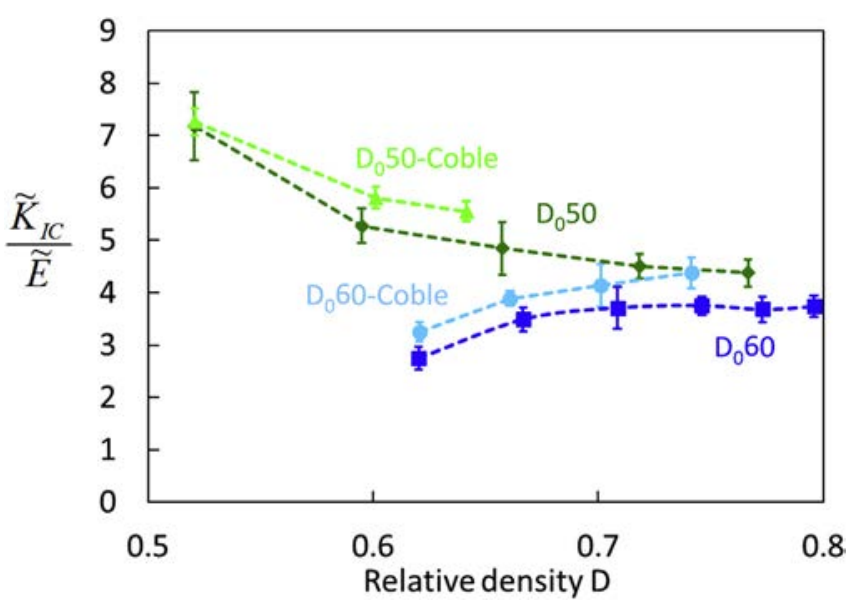

Fig. 7. Strain tolerance parameter $\frac{K^{\sim} \text { Ic }}{E^{\sim}}$ as a function of final relative density for homogeneous samples. Error bars denote the standard deviation of five DEM simulations.

improvement of the $\tilde{K}_{I c} / \tilde{E}$ ratio (Fig. 9b). The gain in Young's modulus obtained by the addition of spherical macropores is explained by larger values of $Z$ and $a_{b} / R$ but also by the superimposition to the microporosity (resulting from partial sintering) of a more mechanically efficient porosity in terms of elasticity [42]. Similarly, in addition to larger necks, the so-called crack-tip blunting effect [43-45] contributes to the fracture toughness increase of the pore former microstructure. Samples obtained from pre-calcined aggregates are very specific in the fact that the intermacropores regions are aggregates of particles already fused by large necks before sintering. This gives rise to a bimodal distribution of neck size as observed in Fig. 8 that will ultimately merge at high relative density. The aggregate microstructure leads to a significant improvement of the fracture toughness due to large necks, but in contrast to the pore former microstructure, the increase of the Young's modulus is not as marked as the increase in fracture toughness, thus leading to the high strain tolerance observed in Fig. 9a. Young's modulus stays relatively low in this case for two main reasons:

- $Z$ is low and similar to the homogeneous microstructure,

- macropores have an irregular shape which is less efficient in terms of elasticity than spherical pores [42].

\subsection{General scaling law}

It is worth noting that the scaling laws (3) and (9), established for homogeneous distribution of $Z$ and $a_{b} / R$, are not appropriate for the heterogeneous microstructures. Indeed, the average values of $Z$ and $a_{b} / R$ cannot describe alone the microstructural complexity of the pore former and aggregate samples, in particular the presence of macropores and the bimodal distribution of neck size of the aggregate microstructure. However, as an attempt to find a very general relationship between $E$ and $K_{I c}$, the scaling laws (3) and (9) can be combined to obtain the following result:

$K_{I c} \propto \frac{E}{Z^{0.9}}\left(\frac{a_{b}}{R}\right)^{0.3} \approx \frac{E}{Z}$

In order to assess the relevancy of this potential simple proportionality relationship, in particular as compared to a simple proportionality between Young's modulus and fracture toughness, $\tilde{K}_{I c}$ is plotted as a function of $\tilde{E}$ and $\tilde{E} / Z$ in Fig. 10 a and Fig. $10 \mathrm{~b}$, respectively. All the numerical samples studied in sections 3.1 and 3.2 are considered, plus two additional samples with bimodal particle size distribution and experimental data on porous alumina from publications by Rodel and co-workers [19,46,47]. Samples with bimodal distributions $D_{0} 48$-bimod and $D_{0} 63$-bimod contained $70 \%$ volume fraction of particles with a size ratio of 2 . The green densities used were 0.48 and 0.63 respectively. The simulation results show that while a simple plot of $\tilde{K}_{I c}$ versus $\tilde{E}$ leads to a large scatter in the data, Eq. (10) is a much better fit to the results for the wide range of numerical microstructures investigated. The proposed law is less striking for experimental data but it should be remembered that there is significant uncertainties in the measurement of mechanical properties and that $Z$ (unreported and probably not accessible on such fine particle system) is estimated using the very simple quadratic law proposed by German [48]. Relation (10) reflects the fact that the variation in $\tilde{K}_{I c} / E$ ratio mainly arises from a variation in $Z$. Nevertheless, a close examination of Fig. 10b indicates that some samples slightly deviate from the master line, due to the fact that other parameters (e.g. the shape of macropores or neck size) also play a second order role. A linear fit of simulation data provides a direct relationship between the mechanical properties of the porous body $\left(E, K_{I C}\right)$, its microstructure $(Z)$ and the properties of the constituent material $\left(E_{0}\right.$ and $\left.\gamma_{s}\right)$ :

$K_{I c} \approx 30 \sqrt{\frac{\gamma_{s}}{E_{0}}} \frac{E}{Z}$

A Poisson's ratio of 0.2 , typical of oxide ceramic materials was assumed to derive Eq. (11). We believe that this relationship can be 
(a)

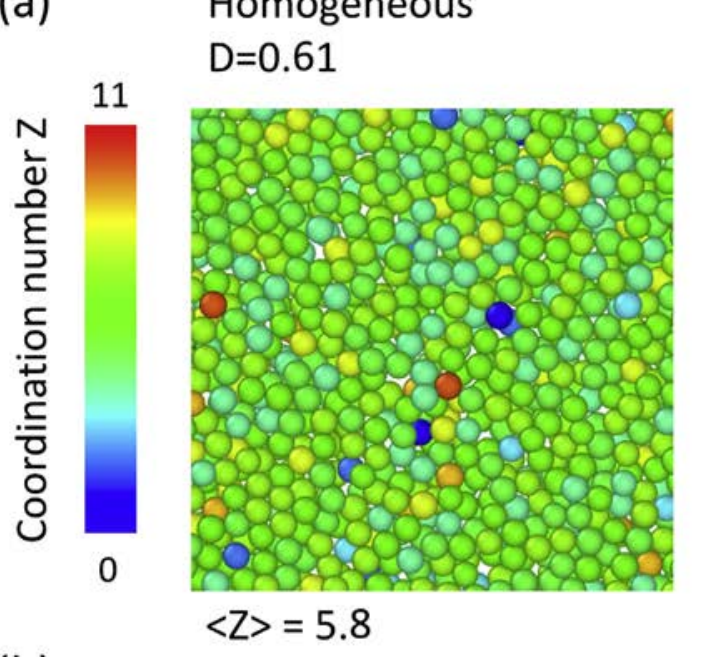

(b)

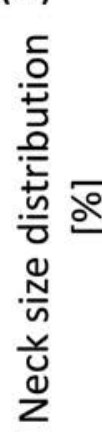
$D=0.61$
Homogeneous

$<\mathrm{Z}>=5.8$

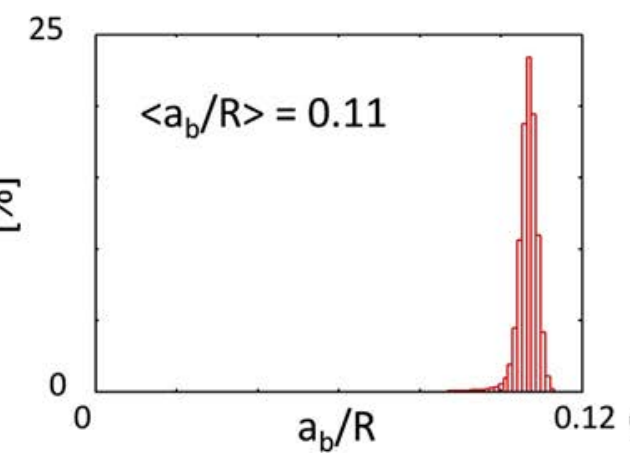

Pore formers

$D=0.61$

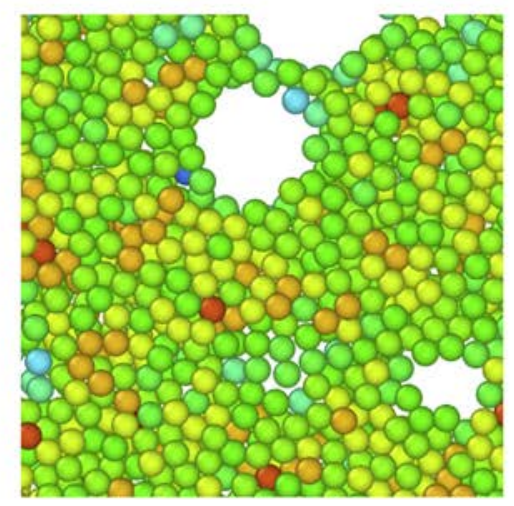

$\langle Z>=6.9$

\section{Aggregates $D=0.61$}

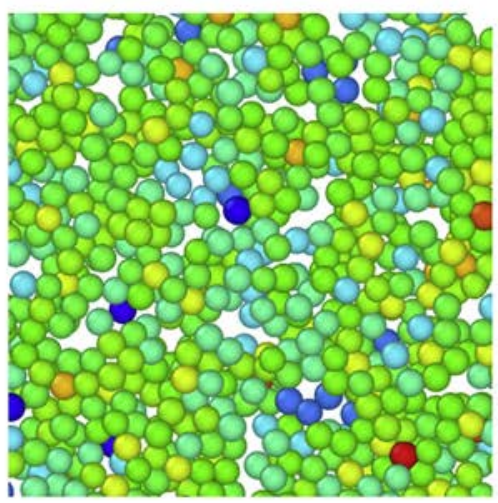

$<Z>=5.9$
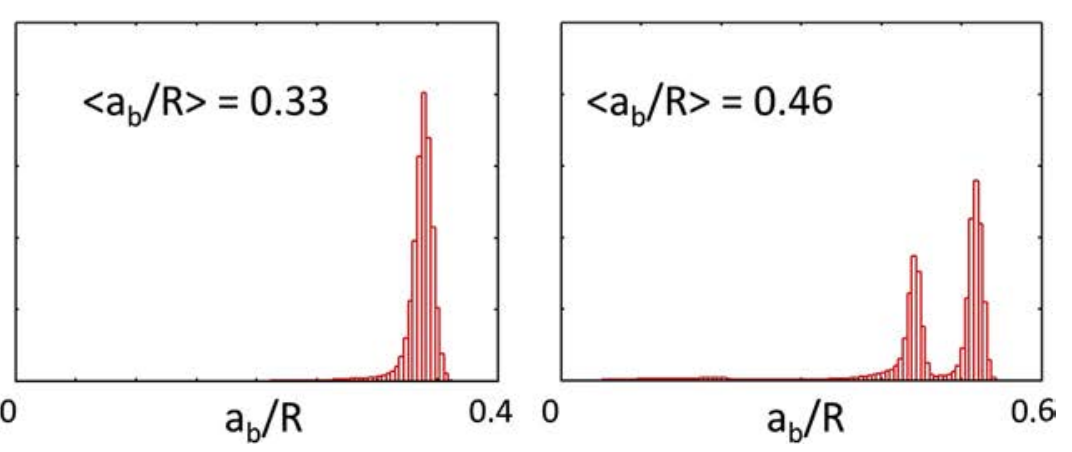

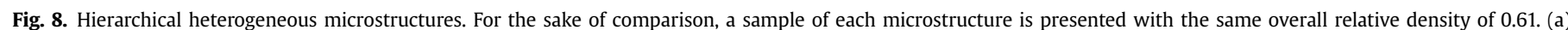
Microstructures and coordination number $Z$. The slices are 3 particles thick. (b) Neck size distribution. Mean values for $\mathrm{a}_{\mathrm{b}} / \mathrm{R}$ and $\mathrm{Z}$ are reported within $<>$.

(a)

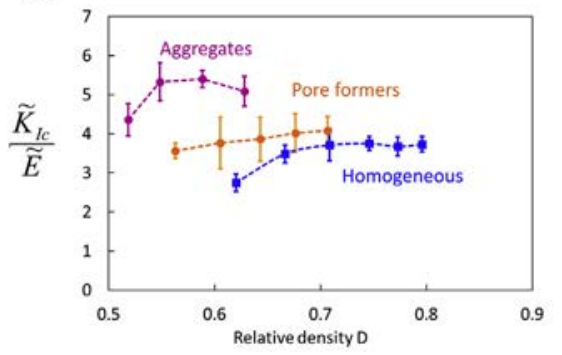

(b)

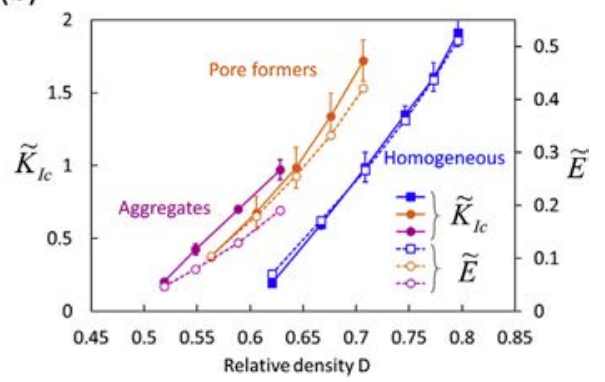

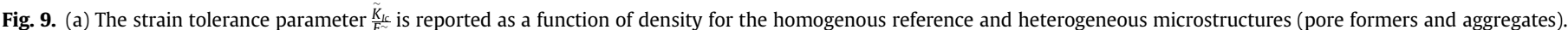

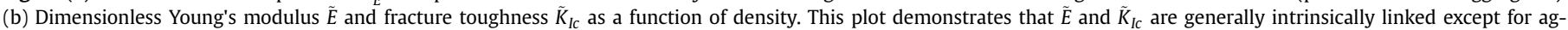
gregates that promote a larger relative increase of $\tilde{K}_{I c}$ compared to $\tilde{E}$. Error bars denote the standard deviation of five DEM simulations.

of significant practical use for material scientists seeking to evaluate and optimize the mechanical properties and particularly strain tolerance of porous ceramic materials or for engineers in designing components and systems with porous ceramics.

\section{Conclusion}

Previous attempts of relating $E$ and $K_{I c}$ of partially sintered materials have been revisited in light of extensive DEM simulations. In particular, scaling laws linking the Young's modulus and the fracture toughness to the microstructure are proposed for any linear elastic partially sintered material. It was found that the proportionality between $E$ and $K_{I c}$ often observed experimentally is related to a more general relationship where the coordination number plays a significant role. The proposed relationship demonstrates that microstructure actually matters for strain tolerant design, in contrast to several previous claims that the strain tolerance parameter $K_{I c} / E$ is independent of the microstructure and density and only dependent on the material [17-20]. In particular starting with low green densities improves the strain tolerance of the material. The introduction of heterogeneity and hierarchy via two different methods has been also evaluated. It turns out that the 
(a)

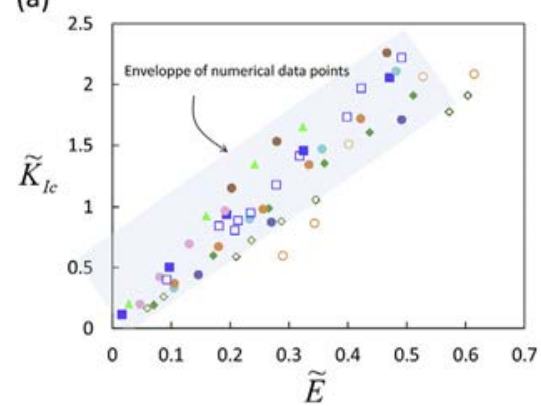

(b)

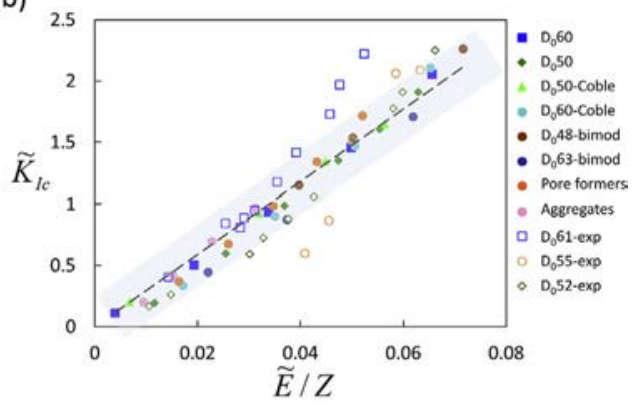

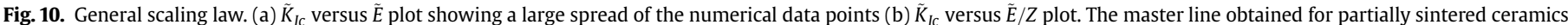

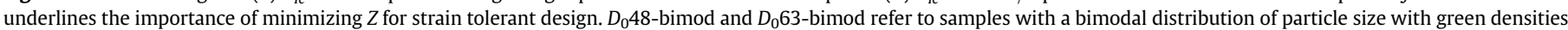

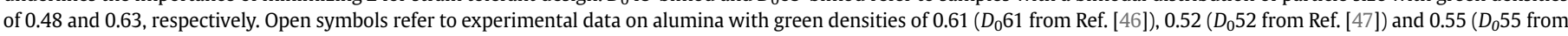
Ref. [19]). (For interpretation of the references to colour in this figure legend, the reader is referred to the Web version of this article.)

use of particle aggregates significantly improves the strain tolerance while the use of spherical macropores, although beneficial, has a more limited effect. Still, these two examples demonstrate that introducing controlled microstructural heterogeneity is an intriguing strategy for improving the mechanical performance of porous materials, especially when considering applications in which failure is caused by differential strains. Finally, the scaling relationship $K_{I c} \propto \frac{E}{Z}$ is proposed and validated by DEM simulations for a wide range of microstructures including hierarchical and bimodal ones.

\section{Acknowledgements}

This research was conducted under the Materials World Network program. Research support by the French Agence Nationale de la Recherche (ANR 2010 BLAN 0931 01) and the US National Science Foundation (grant number DMR-1008600) is gratefully acknowledged.

\section{References}

[1] J. Adler, Ceramic diesel particulate filters, Int. J. Appl. Ceram. Technol. 2 (2005) 429-439, https://doi.org/10.1111/j.1744-7402.2005.02044.x.

[2] Y.W. Kim, Y.J. Jin, J.H. Eom, I.H. Song, H.D. Kim, Engineering porosity in silicon carbide ceramics, J. Mater. Sci. 45 (2010) 2808-2815, https://doi.org/10.1007/ s10853-010-4270-5.

[3] S. Bose, S. Vahabzadeh, A. Bandyopadhyay, Bone tissue engineering using 3D printing, Mater. Today 16 (2013) 496-504, https://doi.org/10.1016/ j.mattod.2013.11.017.

[4] S. Deville, E. Saiz, A.P. Tomsia, Freeze casting of hydroxyapatite scaffolds for bone tissue engineering, Biomaterials 27 (2006) 5480-5489, https://doi.org/ 10.1016/j.biomaterials.2006.06.028.

[5] M. Bohner, G. Baroud, A. Bernstein, N. Do, L. Galea, B. Hesse, R. Heuberger, S. Meille, P. Michel, J. Sague, H. Seeherman, Characterization and distribution of mechanically competent mineralized tissue in micropores of b-tricalcium phosphate bone substitutes, Mater. Today 20 (2017) 106-115, https://doi.org/ 10.1016/j.mattod.2017.02.002.

[6] A. Sarikaya, V. Petrovsky, F. Dogan, Effect of the anode microstructure on the enhanced performance of solid oxide fuel cells, Int. J. Hydrogen Energy 37 (2012) 11370-11377, https://doi.org/10.1016/j.ijhydene.2012.05.007.

[7] T. Suzuki, Z. Hasan, Y. Funahashi, T. Yamaguchi, Y. Fujishiro, M. Awano, Impact of anode microstructure on solid oxide fuel cells, Science 325 (2009) 852-855, https://doi.org/10.1126/science.1176404.

[8] M. Liu, M.E. Lynch, K. Blinn, F.M. Alamgir, Y. Choi, Rational SOFC material design: new advances and tools, Mater. Today 14 (2011) 534-546, https:// doi.org/10.1016/S1369-7021(11)70279-6.

[9] C.M. Doherty, R.A. Caruso, C.J. Drummond, High performance LiFePO4 electrode materials: influence of colloidal particle morphology and porosity on lithium-ion battery power capability, Energy Environ. Sci. 3 (2010) 813 https://doi.org/10.1039/b922898e.

[10] D.J. Green, R. Brezny, C. Nader, The elastic behavior of partially-sintered materials, Mater. Res. Soc. Symp. Proc 119 (1988) 43-48.

[11] D.J. Green, D. Hardy, Fracture toughness of partially-sintered brittle materials, J. Mater. Sci. Lett. 15 (1996) 1167-1168. https://doi.org/10.1007/BF00539970.

[12] P. Arato, E. Besenyei, A. Kele, F. Weber, Mechanical properties in the initial stage of sintering, J. Mater. Sci. 30 (1995) 1863-1871. https://doi.org/10.1007/ BF00351623. (Accessed 3 April 2012).

[13] M.T. McDowell, S.W. Lee, W.D. Nix, Y. Cui, 25th anniversary article: understanding the lithiation of silicon and other alloying anodes for lithium-ion batteries, Adv. Mater. 25 (2013) 4966-4985, https://doi.org/10.1002/ adma.201301795.

[14] J. Cho, Porous Si anode materials for lithium rechargeable batteries, J. Mater Chem. 20 (2010) 4009, https://doi.org/10.1039/b923002e.

[15] J. Christensen, Modeling diffusion-induced stress in Li-Ion cells with porous electrodes, J. Electrochem. Soc. 157 (2010) A366, https://doi.org/10.1149/ 1.3269995.

[16] M.F. Ashby, Materials Selection in Mechanical Design, third ed., Elsevier, 2005.

[17] D. Lam, F.F. Lange, A.G. Evans, Mechanical properties of partially dense alumina produced from powder compacts, J. Am. Ceram. Soc. 77 (1994) 2113-2117, https://doi.org/10.1111/j.1151-2916.1994.tb07105.x.

[18] D. Hardy, D.J. Green, Mechanical properties of a partially sintered alumina, J. Eur. Ceram. Soc. 15 (1995) 769-775, https://doi.org/10.1016/0955-2219(95) 00045-V.

[19] B.D. Flinn, R.K. Bordia, A. Zimmermann, J. Rödel, Evolution of defect size and strength of porous alumina during sintering, J. Eur. Ceram. Soc. 20 (2000) 2561-2568. S0955221900001333.

[20] A. Atkinson, P. Bastid, Q. Liu, Mechanical properties of magnesia-spinel composites, J. Am. Ceram. Soc. 90 (2007) 2489-2496, https://doi.org/ 10.1111/j.1551-2916.2007.01733.x.

[21] D. Jauffrès, C.L. Martin, A. Lichtner, R.K. Bordia, Simulation of the elastic properties of porous ceramics with realistic microstructure, Model. Simulat. Mater. Sci. Eng. 20 (2012) 45009, https://doi.org/10.1088/0965-0393/20/4/ 045009.

[22] D. Jauffres, C.L. Martin, A. Lichtner, R.K. Bordia, Simulation of the toughness of partially sintered ceramics with realistic microstructures, Acta Mater. 60 (2012) $4685 . \quad$ http://www.sciencedirect.com/science/article/pii/ S1359645412003345.

[23] C.L. Martin, R.K. Bordia, Influence of adhesion and friction on the geometry of packings of spherical particles, Phys. Rev. E 77 (2008) 31307.

[24] D. Bouvard, R.M. McMeeking, Deformation of interparticle necks by diffusioncontrolled creep, J. Am. Ceram. Soc. 79 (1996) 666-672, https://doi.org 10.1111/j.1151-2916.1996.tb07927.x.

[25] C.L. Martin, R.K. Bordia, The effect of a substrate on the sintering of constrained films, Acta Mater. 57 (2009) 549-558. http://www.sciencedirect. com/science/article/pii/S135964540800699X.

[26] R.M. McMeeking, G. Jefferson, G.K. Harritos, Elastic and Visco-elastic response of finite particle junctions in granular materials, in: A. Zavalianglos A;Laptev (Ed.), Recent Dev. Comput. Model. Powder Metall. Process, IOS Press, Amsterdam, 2001, pp. 50-62.

[27] G. Jefferson, G.K. Haritos, R.M. Mcmeeking, G. Je, G.K. Haritos, R.M. Mcmeeking, The elastic response of a cohesive aggregate-a discrete element model with coupled particle interaction, J. Mech. Phys. Solid. 50 (2002) 2539-2575. http://www.sciencedirect.com/science/article/B6TXB46XGKR7-2/2/eff9a645b11419d1923b63f12fdb9f60.

[28] D. Potyondy, P. Cundall, A bonded-particle model for rock, Int. J. Rock Mech. Min. Sci. 41 (2004) 1329-1364, https://doi.org/10.1016/j.ijrmms.2004.09.011.

[29] H. Fujita, G. Jefferson, R.M. Mcmeeking, F.W. Zok, Mullite/alumina mixtures for use as porous matrices in oxide fiber composites, J. Am. Ceram. Soc. 87 (2004) 261-267.

[30] X. Liu, C.L. Martin, D. Bouvard, S. Di Iorio, J. Laurencin, G. Delette, Strength of highly porous ceramic electrodes, J. Am. Ceram. Soc. 94 (2011) 3500-3508, https://doi.org/10.1111/j.1551-2916.2011.04669.x.

[31] R.L. Coble, Initial sintering of alumina and hematite, J. Am. Ceram. Soc. 41 (1958) 55-62, https://doi.org/10.1111/j.1151-2916.1958.tb13519.x.

[32] E. Artz, The influence of an increasing particle coordination on the 
densification of spherical powders, Acta Metall. 30 (1982) 1883-1890.

[33] W. Luo, J. Pan, Effects of surface diffusion and heating rate on first-stage sintering that densifies by grain-boundary diffusion, J. Am. Ceram. Soc. 98 (2015) 3483-3489, https://doi.org/10.1111/jace.13662.

[34] S. Oh, K. Tajima, M. Ando, T. Ohji, Fabrication of porous Al 203 by microwave sintering and its properties, Mater. Lett. (2001) 2-5.

[35] D. Doni Jayaseelan, N. Kondo, M.E. Brito, T. Ohj, High-strength porous alumina by the pulse electric current sintering technique, J. Am. Ceram. Soc. 85 (2002) 267-269.

[36] F. Zhang, K. Lin, J. Chang, J. Lu, C. Ning, Spark plasma sintering of macroporous calcium phosphate scaffolds from nanocrystalline powders, J. Eur. Ceram. Soc. 28 (2008) 539-545. http://www.sciencedirect.com/science/article/B6TX0 4PNF9T7-1/2/0ae51cd751cede60c78ff79e0f664a67.

[37] S. Perko, A. Dakskobler, T. Kosmac, High-performance porous nanostructured ceramics, J. Am. Ceram. Soc. 93 (2010) 2499-2502, https://doi.org/10.1111/ j.1551-2916.2010.03835.x.

[38] A. Kritikaki, A. Tsetsekou, Fabrication of porous alumina ceramics from powder mixtures with sol-gel derived nanometer alumina: effect of mixing method, J. Eur. Ceram. Soc. 29 (2009) 1603-1611, https://doi.org/10.1016 j.jeurceramsoc.2008.10.011.

[39] C. Liao, T. Chang, D. Young, Stress-strain relationship for granular materials based on the hypothesis of best fit, Int. J. Solid Struct. 34 (1997) 4087-4100.

[40] X. Liu, C.L. Martin, G. Delette, D. Bouvard, Elasticity and strength of partially sintered ceramics, J. Mech. Phys. Solid. 58 (2010) 829-842, https://doi.org/ 10.1016/j.jmps.2010.04.007
[41] D. Simwonis, H. Thülen, F.J. Dias, A. Naoumidis, D. Stöver, Properties of Ni/YSZ porous cermets for SOFC anode substrates prepared by tape casting and coatmix process, J. Mater. Process. Technol. 92-93 (1999) 107-111.

[42] A.P. Roberts, E.J. Garboczi, Elastic properties of model porous ceramics, J. Am. Ceram. Soc. 83 (2000) 3041-3048. https://doi.org/10.1111/j.1151-2916.2000. tb01680.x.

[43] Z.-Y. Deng, J. She, Y. Inagaki, J.-F. Yang, T. Ohji, Y. Tanaka, Reinforcement by crack-tip blunting in porous ceramics, J. Eur. Ceram. Soc. 24 (2004) 2055-2059, https://doi.org/10.1016/S0955-2219(03)00365-0.

[44] M. Ryvkin, J. Aboudi, Crack resistance in two-dimensional periodic materials of medium and low porosity, Eng. Fract. Mech. 78 (2011) 2153-2160, https:// doi.org/10.1016/j.engfracmech.2011.04.004.

[45] N. Fleck, X. Qiu, The damage tolerance of elastic-brittle, two-dimensional isotropic lattices, J. Mech. Phys. Solid. 55 (2007) 562-588, https://doi.org/ 10.1016/j.jmps.2006.08.004.

[46] T. Ostrowski, A. Ziegler, R.K. Bordia, J. Rödel, Evolution of Young's modulus, strength, and microstructure during liquid-phase sintering, J. Am. Ceram. Soc. 81 (1998) 1852-1860.

[47] T. Ostrowski, J. Rodel, Evolution of mechanical properties of porous alumina during free sintering and hot pressing, J. Am. Ceram. Soc. 82 (1999) 3080-3086, https://doi.org/10.1111/j.1151-2916.1999.tb02206.x.

[48] R.M. German, Coordination number changes during powder densification, Powder Technol. 253 (2014) 368-376, https://doi.org/10.1016/ j.powtec.2013.12.006. 